Cahiers de recherches médiévales

Journal of medieval studies

$8 \mid 2001$

La protection spirituelle au Moyen Âge

\title{
Sainte Catherine, Jeanne d'Arc et le « saut de Beaurevoir »
}

\section{Françoise Michaud-Fréjaville}

\section{(2) OpenEdition}

Édition électronique

URL : https://journals.openedition.org/crm/386

DOI : $10.4000 / \mathrm{crm} .386$

ISSN : 1955-2424

Éditeur

Honoré Champion

\section{Édition imprimée}

Date de publication : 15 janvier 2001

Pagination : 73-86

ISSN : $1272-9752$

\section{Référence électronique}

Françoise Michaud-Fréjaville, "Sainte Catherine, Jeanne d'Arc et le « saut de Beaurevoir » », Cahiers de recherches médiévales [En ligne], 8| 2001, mis en ligne le 13 mars 2008, consulté le 15 décembre 2022. URL : http://journals.openedition.org/crm/386 ; DOl : https://doi.org/10.4000/crm.386

Ce document a été généré automatiquement le 15 décembre 2022.

Tous droits réservés 


\title{
Sainte Catherine, Jeanne d'Arc et le "saut de Beaurevoir »
}

\author{
Françoise Michaud-Fréjaville
}

1 Les « voix » de Jeanne d'Arc ont eu de très lointains échos. Ceux qui «y croient » et ceux qui «n'y croient pas » continuent à s'opposer, moins violemment certes qu'au moment de l'affaire Thalamas au début du siècle dernier, mais néanmoins sans concessions ${ }^{1}$. On sait que la médecine et la psychiatrie s'en sont mêlées pour déterminer une origine pathologique aux manifestations appelées "voix, visions", sans éclairer quiconque ${ }^{2}$. Après que les peintres aient abandonné l'évocation des anges évanescents et des saintes transparentes, on a vu récemment réapparaître à l'écran les trucages d'éclairs et de nuages, les surimpressions bleutées, les brumes fantomatiques qui firent les belles heures des projections de Georges Méliès ${ }^{3}$. Dans tout cela sont oubliés et la période où se place l'aventure de Jeanne et ce qu'on pensait et attendait au début $\mathrm{du} \mathrm{XV}^{\mathrm{e}}$ siècle de l'intervention des anges et des saints auprès des humains. On passe aussi bien trop facilement sur ce qui a été réellement dit lors des procès et plus particulièrement lors du procès de condamnation pour reproduire ce qu'en ont conservé les historiens de tous bords, et pour monter en épingle ce qu'ont bien voulu retenir de leurs lectures de seconde main les pseudo chercheurs, les hagiographes, les romanciers, les scénaristes, plus d'ailleurs que les poètes.

2 Un des épisodes rarement évoqué par l'histoire populaire de Jeanne parce que considéré comme non exemplaire, voire même réellement peu édifiant pour la jeunesse, est celui de ses tentatives d'évasion des châteaux de Beaulieu et de Beaurevoir où elle fut enfermée entre la prise à Compiègne et le procès à Rouen, de mai à novembre 1430. Même les auteurs les plus favorables à l'héroïne paraissent avoir été influencés par les arguments des juges de Rouen qui y virent tout à tour une preuve de désespoir, une tentative de suicide, une désobéissance envers les voix et finalement un défi à Dieu et, pour paraphraser Jeanne, les historiens passent outre. Je voudrais reprendre ce dossier et tenter de relier les deux essais que fit la prisonnière pour s'enfuir de Beaulieu et de Beaurevoir, avec ce qui se savait dans le monde qu'elle avait connu du rôle particulier de sainte Catherine dans la libération miraculeuse des 
prisonniers. Le Livre des miracles de Sainte-Catherine de Fierbois ${ }^{4}$ et le texte du Procès de condamnation permettront peut-être d'y voir un peu plus clair. De façon pour nous très intéressante, ce sont finalement les questions sans indulgence du tribunal auxquelles la jeune fille fut confrontée qui lui ont peut-être permis d'analyser ses propres relations avec ses voix et d'accepter que sainte Catherine n'ait pas accompli pour elle ce que la sainte passait pour avoir réalisé - et ferait encore - pour d'autres.

3 Nous n'aborderons pas ici à fond la question de savoir quand Jeanne mit un nom sur les voix ${ }^{5}$. On sait qu'elles ne sont réellement personnalisées dans le texte du procès de condamnation que vers la fin de la séance du mardi 27 février 1431: «Interrogée si c'était la voix d'un ange qui lui parlait ou si c'était la voix d'un saint ou d'une sainte, ou celle de Dieu directement, elle répondit que cette voix était celle de sainte Catherine et de sainte Marguerite. Et leur figures sont couronnées de belles couronnes très riches et très précieuses ${ }^{6} »$. Jeanne ajouta qu'elle avait déjà parlé de cela (de hoc) lors de l'interrogatoire de Poitiers, sans qu'on puisse déterminer aujourd'hui s'il s'agissait des voix ou des individualités des saintes, et continua en affirmant qu'elle savait parfaitement les distinguer l'une de l'autre (bene cognoscit unam ab altera) et finalement affirma que c'est par elles-mêmes qu'elle sut leur nom (per hoc cognoscit quod se nominant ei). Pressée de questions sur leurs vêtements, leur âge, la première qui lui apparut, leurs discours simultanés elle rétorqua "Je n'ai pas le droit de parler de ça ${ }^{7}$ ». Au long du procès les juges s'intéressèrent davantage à saint Michel, à son rôle dans le message que la Pucelle devait délivrer au roi, qu'aux saintes, mais il est révélateur, à mon avis, que la voix individualisée de Catherine soit celle dont elle évoque le réconfort après l'échec de l'évasion de Beaurevoir : « La voix de sainte Catherine lui dit quelle fît bonne figure et qu'elle guérirait ${ }^{8}$ ». La sainte est constamment, on le verra, évoquée lorsque affaire revient dans les interrogatoires et les conclusions. Catherine était la sainte des évasions réussies et son sanctuaire de Fierbois, à la limite de la Touraine et du Poitou, était réputé chez les partisans de Charles VII pour l'efficacité des prières que lui adressaient du fond de leur geôle les malheureux captifs.

4 Le Livre des Miracles fut compilé en français après 1470 peut-être pour la reine Charlotte de Savoie (†1483), mais les deux cent trente-sept documents auxquels ont eu accès les rédacteurs pour les recueillir ont dû s'accumuler dès la fin du XIV ${ }^{e}$ siècle dans les archives de la chapelle tourangelle. Les ex-voto et les récits qui couraient dans le village et sans doute jusqu'à Tours, les anecdotes que l'on contait aux camps des armées armagnacques en déplacement, les explications des bénéficiaires eux-mêmes que l'on pouvait encore rencontrer entretenaient déjà au temps de Jeanne la certitude de l'efficacité des prières à la sainte.

5 La popularité du sanctuaire, dans un écart méridional de la paroisse de Sainte-Maure à une journée de marche de Tours, après son «invention » en 1375 par un habitant des environs a tout d'abord, si l'on suit la chronologie du Livre des Miracles, été liée à ses vertus thaumaturgiques. L'introduction du Livre dit clairement que «le voyage de madame sainte Catherine [...] fut trouvé par un prodomme dudit lieu que l'on appelle Jehan Godefroy $»^{10}$, Jean désespérant de guérir se souvint en 1375 d'une chapelle en ruine de la paroisse, s'y fit porter et "fut sains et gueri de tous ses membres", inaugurant ainsi le pèlerinage de demande, «le voyage». Le quatrième miracle enregistré par le recueil offre le premier vœu à distance (depuis l'Angoumois) suivi d'oblation $^{11}$, le cinquième semble montrer que la réputation du lieu rayonne en 1379 également jusqu'à Angers ${ }^{12}$, et le sixième signale déjà un sanctuaire nécessitant des 
travaux ${ }^{13}$. Les vingt-sept premiers miracles (1375-1379), sont tous des guérisons inespérées de maladie ou des issues heureuses d'accidents gravissimes après un vœu des victimes ou de leurs proches de se rendre à la chapelle ou d'y faire une oblation. Jusqu'ici il n'y a guère d'originalité qui distingue particulièrement cette sainte Catherine et son sanctuaire de Fierbois des autres endroits favorisés par des miracles, sinon le nombre important de ceux-ci en un laps de temps fort court et, par conséquent, un attrait qui ne pouvait que croître. Le grand nombre de faits sans date ou de localisation imprécise et les incohérences chronologiques des quelques actes datés empêchent cependant d'examiner les voies par lesquelles se transmirent si vite les nouvelles de l'efficacité du lieu.

6 Le samedi 12 mai 1380 Pierre Chappon vint rendre grâce pour sa délivrance miraculeuse $\mathrm{e}^{14}$. Vingt-huitième enregistré c'est le troisième acte exactement daté du recueil. Il porte déjà une partie des caractéristiques de la protection particulière de la sainte envers les prisonniers et en même temps ce texte précis provoque quelques doutes: Pierre Chappon, pris par les Anglais et mis au fers un mois entier, s'est retrouvé un matin, après s'être endormi en faisant le vœu de se rendre à la chapelle de Sainte-Catherine de Fierbois, chez lui " en sa salle », toujours enferré. On a là un des éléments essentiels des manifestations de la protection de Catherine : le prisonnier, injustement enfermé n'intervient pas ou peu dans son élargissement, il devient invisible et se transporte dans le temps et l'espace, sans conscience. L'étrangeté de cette délivrance pour nos yeux bien rationalistes, s'accroît ici de la conjonction d'une prière identique de sa femme "environ cette heure " et l'éloignement spatial du sanctuaire tourangeau d'une affaire qui s'est déroulée du côté de Senlis ${ }^{15}$. La date du texte doit être celle du pèlerinage accompli en Touraine. On doit néanmoins se demander si Fierbois ne fut pas alors une simple étape car le texte sépare le vœu à sainte Catherine et la mention d'une chapelle non vraiment localisée « il se rendrait à la chapelle de céans ", au long d'un périple des sanctuaires et chapelles de la sainte par Paris, Mantes, Rouen et autres lieux favorisés de sa protection.

7 Catherine était en effet honorée depuis longtemps à Rouen, la ville passait jusqu'à la fin $\mathrm{du} \mathrm{XIV}^{\mathrm{e}}$ siècle pour être le seul lieu en dehors du Sinaï où se trouvaient des reliques de la jeune martyre: elles en auraient été rapportées au tout début du $\mathrm{XI}^{\mathrm{e}}$ siècle $^{16}$ et déposées à l'abbaye de la Trinité-des-Monts lors d'un grand mouvement de collecte de reliques venues de la Chrétienté tout entière, pour la plus grande gloire des ducs qui utilisaient ainsi leurs relations avec le monde normand méditerranéen ${ }^{17}$. La Légende dorée rapporte ainsi qu'à la demande d'un moine de Rouen un doigt de la sainte se détacha de lui-même du corps gisant dans son tombeau ${ }^{18}$. En revanche, la présence de reliques à Fierbois n'est pas assurée du tout à la date de 1380, alors que le culte général de la sainte est devenu assez populaire en Occident, attesté par les patronages de confréries $^{19}$, les copies de sa $v^{2}{ }^{20}$, les heures en son honneur ${ }^{21}$, les statues, les peintures $^{22}$. On pense que leur arrivée pourrait être un des éléments de la dévotion et protection que Jean II le Meingre dit Boucicaut (v. 1366-1421), maréchal de France, manifesta envers le sanctuaire de Fierbois. Au cours d'une première expédition plutôt pacifique en Orient en 1389, il se serait rendu au monastère du Mont-Sinaï et aurait peut-être obtenu, à son tour, une phalange de la sainte ${ }^{23}$. Une autre occasion, plus vraisemblable à mon sens, aurait pu être le voyage de Constantinople en 1400 d'où il aurait rapporté un fragment de cette sainte dont la protection lui aurait permis de survivre au désastre de Nicopolis (1396) et de rentrer de captivité avec tous les honneurs dû à une bravoure déjà légendaire ${ }^{24}$. En tous cas, il fit tout pour lier le lieu où 
des miracles avaient déjà manifesté le pouvoir d'intercession de la sainte, venue du pays Mamelouk et qui avait résisté au roi d'Égypte, au chemin vers Saint-Jacques de Compostelle où l'on venait prier le Matamore, l'intercesseur qui se serait porté en personne au secours des Chrétiens contre les Musulmans à la bataille de Clavijo (844). Avant 1408 , le maréchal devenu le "persécuteur des mescreans ${ }^{25}$ avait fondé une aumônerie avec une chapelle dédiée à saint Jacques dans le village de Sainte-Catherine de Fierbois, halte suffisamment fréquentée pour que le pape Benoît XIII autorisât le 26 juin 1408 l'implantation d'un cimetière afin d'éviter d'avoir à faire les inhumations à Sainte-Maure, chef-lieu de la paroisse ${ }^{26}$. Le renom «militaire» de la chapelle de la sainte en reçut un surplus de reconnaissance : entre 1383 et 1400 il n'y eut que quatre délivrances miraculeuses de prisonniers pour vingt-deux miracles de guérisons, moins du cinquième, mais à partir de 1400 et jusqu'en mai 1430 - notre date butoir pour cette étude - on en compte vingt-six, près de la moitié ( $44 \%)$ des miracles répertoriés et datables qui eurent lieu au cours de cette génération. Ils concernèrent quarante-six personnes qui toutes cependant ne sont pas nommées ni ne firent le voyage d'action de grâce. Que le généreux et vaillant maréchal ait été un des captifs survivants du désastre d'Azincourt et qu'il soit mort en Angleterre en 1421, toujours prisonnier donc sans une intervention miraculeuse peut-être ardemment invoquée, ne semble pas avoir eu d'influence négative pour Fierbois. Au contraire, la perte de la Normandie en 1418 et l'impossibilité d'accéder au sanctuaire de Rouen, ville anglaise depuis janvier 1419, et à son fragment de dépouille (pourtant authentifiée par l'autorité de la Légende dorée), semblent avoir fort accru le pouvoir de la chapelle tourangelle aux yeux des partisans de Charles VII qui disposaient ainsi d'une protection de la sainte, rigoureusement parallèle et tout aussi valable. De 1418 à la capture de Jeanne d'Arc en mai 1430, seize libérations (les deux tiers de celles de la période) dues à l'intercession expresse de sainte Catherine ont fait l'objet de notices à Fierbois.

8 Les relations qui en furent compilées dans le Livre des Miracles présentent un certain nombre de caractères qui permettent, à mon avis, d'établir des relations avec les épisodes des tentatives d'évasion de Jeanne d'Arc des châteaux de Picardie au cours de l'été de 1430.

9 Les bénéficiaires, à partir de 1400 , sont en alternance de vrais militaires, chevaliers ${ }^{27}$, gentilshommes ${ }^{28}$, écuyers ${ }^{29}$, gens de garnison ${ }^{30}$, mercenaires suisse ${ }^{31}$ ou écossais ${ }^{32}$ sur lesquels nous reviendrons et de pauvres gens pris par hasard, comme ces quatre Marchois de La Souterraine qui, sans doute dans les années 1380, étaient sortis sans mal d'un rapt commis par des Anglais ${ }^{33}$, ou Clément de la Brière, aussi prisonnier des Anglais, et dont les liens se défirent en vue de Chalucet en Limousin, avant que les ennemis aient pu le mettre en geôle ${ }^{34}$ ou enfin Alain Le Roux de Montreuil-Bellay, miraculeusement délié pendant le sommeil de plomb de ses geôliers ${ }^{35}$. La protection de la sainte répond à la fois aux demandes des soldats prisonniers dont les geôliers ou « les maitres » ne suivent pas formellement les lois de la guerre et aux prières des victimes sans armes des ennemis, voire des amis, lesquels menacent de les mettre injustement aux fers ou à rançon. Ces ennemis sont quatre fois sur cinq des Anglais, présents partout autour de la Touraine : ceux du Limousin en 1383-1384, de Normandie et du Maine, à Falaise en 1418, à Verneuil du Perche en 1420, à Bellême en 1425, à Alençon en 1426, ceux de Beauce en $1426^{36}$. Mais on trouve également des Bourguignons à Beaumont-sur-Oise en 1418, à Corbeil en 1420 et puis encore des Bretons près de Clisson en 1429, des Flamands à Poperinghe en 1382, et même des alliés Écossais qui en 1423 
voulurent se venger de «brigands" sur d'innocents berrichons dont la sainte exauça les prières ${ }^{37}$.

10 Pris, militaires, voyageurs ou paysans sont ligotés étroitement à trois tours attachés à un cheval « les piez par dessoubz le ventre $»^{38}$, enfermés dans « une citerne[...] quinze semaines sans manger et sans boire $\aleph^{39}$, immobilisés "tout en haut de la tour, en fers $»^{40}$, bloqués derrière «troys fortes barres pour barrer l'huys », ou enterrés « en une étroite fosse de la hauteur d'une lance, et pardessus une très grosse soche $»^{41}$, enfin glissés à l'intérieur d'une " pipe sur bout [...] deux grand tables dessus et un Anglois couchié dessus lesdites tables ${ }^{42}$. Il était nécessaire que l'immobilisation soit décrite comme si complète et les liens si infrangibles que seule une intervention extérieure et miraculeuse puisse expliquer que les barres glissent " comme si ce fut un baston ", que les tables se disjoignent pendant la nuit avec à côté "l'Anglois a terre endormy ", que la corde qui lie la huche-prison se rompe ${ }^{43}$ et surtout que l'on se retrouve libre auprès de la «soche remuée ainsi comme deux tours", que les liens tombent des mains et des pieds. Il faut reconnaitre que le miracle du transfert de la prison au coin du feu conjugal n'a eu lieu qu'une fois, pour Perrot Chappon déjà rencontré. Dans les autres cas le prisonnier doit généralement subir encore quelques épreuves sur lesquelles nous devrons revenir, toujours sous l'égide protectrice de la sainte, jusqu'à l'accomplissement du vœu : se rendre à la chapelle de Fierbois.

11 Les captures exécutées, tout au moins en apparence, hors du combat proprement guerrier sont cependant suivies de demandes ou de menaces de rançon, ce qui n'est guère légitime. Un homme d'Aunis en a payé la moitié mais, à bout de ressources «ne peut finer de l'autre moitié $»^{44}$. Des cinq compagnons mis sous la souche et qui ne pouvaient s'engager à verser une « si grosse ranson que tous leurs amys eussent eu trop a faire de la payer" trois moururent au bout de quatorze mois et trois jours. La défection de son compagnon d'infortune, libéré de Rouen pour ramener les sommes exigées, a poussé Guillaume à se tourner vers la vierge Catherine ${ }^{45}$. Pour les hommes voués à la guerre, les rançons fixées sont disproportionnées : Guillem Guy doit choisir entre payer cinquante écus ou faire libérer deux Anglais ${ }^{46}$, Baudet Lalemand se voit demander littéralement la bourse (trois cents écus) ou la vie et « jura que pour mourir il ne pourroit payer vingt escus ». Ces excès des ennemis justifient aussi que les prières des prisonniers trouvent en Catherine, elle aussi injustement accusée et mise en prison, une bienveillante attention. En outre, même en dehors des liens ou des fers et de la prison étroite, les conditions de détention sont indignes : le pain et l'eau pendant douze semaines pour Girart Redon, trompille de Jean $\mathrm{V}$ de Bretagne ${ }^{47}$, quatorze mois (et trois jours...) de jeûne semblable infligés à Thomas du Mont et Perrinet l'Auvergnat à Corbeil. Enfin l'ennemi impose une durée inusitée des réclusions : trois ans et demi et quatre ans dans les fers de Bellême à deux paroissiens d'Argentré (Jean Ducoudray et Jean Courtin $)^{48}$. À cela il faut ajouter les menaces de " mourir villainement » adressées à l'écuyer Baudet Lalement, la mort sous ses yeux, « et estoit lié au pied du gibet », de ses six compagnons pour le gentilhomme Jean Godelin ${ }^{49}$, et le supplice de ses sept compatriotes berrichons devant Jean de Pons Prins. De la menace l'ennemi est passé à l'acte: Michel Amiclon fut bel et bien pendu «en chemise $»^{50}$, et Pierre Dufons fut même dûment enseveli ${ }^{51}$. Épuisé pour avoir couru à pied «à l'erre de leur cheval », Jean Hurpois n'aspire qu'à mourir, ses bourreaux ont déjà trouvé le lieu, l'un d'eux a tiré l'épée de l'exécution et frappé. 

d'intercession. Le Livre des miracles reste dans la stricte orthodoxie: la prière du quémandeur est portée par la vierge martyre à Dieu, elle demande que ce qui lui fut accordé à elle, c'est-à-dire le réconfort dans la prison, la protection contre les mauvais traitements et les supplices et même la translation de son corps, soit donné aussi aux malheureux enfermés. Du fond de sa huche, Casin se remémore les « vertuz et miracles que Dieu faisoit a la requeste de la glorieuse vierge madame Katherine [...] en luy suppliant quelle pleust estre a son aide envers Dieu». Baudet Lalemant à genoux prie : "Ma très souveraine Dame, je suis certain que tu as puissance envers Dieu mon createur de ayder a tous ceulx et celles qui devotement et de bon cueur te requierent $"$. Montant au gibet, à la troisième marche Pierre de Saint-Jean se souvint « de Dieu et de madame sainte Katherine de Fierboys. Et par grant devocion il va prier et requerre qu'il lui pleust que il ne mourist point de si vilaine mort comme d'estre pendu sans cause ${ }^{52}$ ». Thomas du Mont et Perrinet l'Auvergnat se mirent à genoux « le visage le plus droit qu'ilz purent envers la chapelle de madame sainte Katherine de Fierboys ». Les rudes spécialistes de la guerre et des camps se jurent de faire de durs sacrifices : rassemblés en une même prière le chevalier Jean des Croix, son frère, son neveu et un écuyer promettent de ne plus boire que de l'eau tant qu'ils n'auront pas été à Fierbois remercier la sainte si son intervention auprès de Dieu les sauve du passage en Angleterre $^{53}$, Baudet Lalemant avait précisé de son côté qu'il ne boirait pas de vin jusqu'à l'accomplissement de son vœu d'aller à la chapelle. Le pain et l'eau, parfois tout $\mathrm{nu}$, c'est à dire en chemise, au long du chemin vers le sanctuaire est, on le sait, une des promesses du récit de miracle à travers toute la Chrétienté.

Nous ne saurons rien des demandes sans réponses, des vœux non accomplis, des âmes endurcies et ingrates. En revanche nous apprenons comment les évadés reconnaissants ont pu quitter leur prison. D'abord, les prisonniers recouvrent la liberté de gestes : les cordes de Clément de la Biere "churent à terre »; les liens des quatre hommes de la Souterraine "leur churent des mains et des pieds » ${ }^{54}$; Perrin le Maçon et ses deux compagnons «estoient tous liez: et leur veu fait, ilz se trouverent desliez ${ }^{55}$; étroitement garottés de trois cordes Perrin Gougeon et Moricet Girart se sentent subitement libres ${ }^{56}$. Réputés plus solides encore, les fers ne résistent pas mieux au pouvoir de la sainte : «les septs se ouvrirent et lui churent les fers des jambes », et le prisonnier de la Tour de Broue se précipita vers la liberté ${ }^{57}$. Pierre la Coudre dut y mettre du sien mais se déferra sans peine ; François Dye et Jean Gilbert réussirent à se déferrer les mains mais durent se sauver « encouplez ensemble en une paire de fers " ${ }^{5}$. Jean Ducoudray et ses compagnons, forts de leur vœu, arrivèrent les uns à se déferrer, les autres à sortir des seps ${ }^{59}$. Une fois recouvrée la liberté de mouvements, le miracle perdure par la facilité avec laquelle portes et murs sont franchis : les hommes de la Souterraine trouvèrent les portes ouvertes, les hommes d'armes qui les avaient pris ne les reconnurent pas et les laissèrent passer. Casin du Boys est sorti de sa huche sans que se soient éveillés ni celui qui l'avait pris ni sa «chambrière ». Ne sortirent pas plus du sommeil les geôliers de Baudet Lalemant. La sainte aide parfois à franchir les obstacles par un soutien physique direct : la hauteur de l'appui des fenêtres (deux hommes) n'est rien pour Casin car il « fut advis qu'on le sourdit par dessoubs les esselles et adonc s'en yssit hors de la maison »; François Dye et Jean Gilbert se jettent du haut d'un mur d'une lance et demi de haut sans se faire aucun mal ; Alain le Roux est devenu invisible dans le bois où ses ennemis le cherchent activement ${ }^{60}$ et nous savons déjà que Perrot Chappon s'est endormi enferré à Chamat et réveillé, toujours entravé certes, mais chez 
lui à Luzarches. La sainte sait ranimer aussi bellement l'initiative et l'audace, « après ce qu'il eust fait son veu, sa priere, tout le cueur lui va alleger et assurer " et l'écuyer André Estorneau, dont les pieds étaient liés sous le cheval, se débarrasse de son escorte ennemie, se détache en se renversant sous sa monture, et trouve son chemin dans la nuit et brave l'inconnu des rivières en crue, par la « grace de Dieu et à la requeste de la glorieuse vierge madame sainte Katherine ". Le chevalier Jean des Croix et sa petite mesnie en fuite, arrivés devant un fossé trop large et trop profond à leurs yeux, demandent derechef à genoux l'aide de Catherine, et aussitôt « leur fut advis que l'eau fut apeticee » : ils n'en avaient désormais pas plus haut que les genoux. Il apparait bien dans ces deux derniers cas que les nobles soldats sont tenus de trouver en eux-mêmes le surplus de cœur pour participer à leur propre sauvetage.

Des récits attestent de la certitude des prisonniers que sans l'aide tangible de sainte Catherine ils seraient encore aux fers, ou pire morts. Pour Jean de Pons Prins, avant que la corde casse, il «lui sembloit qu'on le soutenoit par les pieds», aussi n'eut -il pas peur ${ }^{61}$, de plus il se reçut ensuite sur un tas de cailloux bien pointus et « et oncques ne lui firent mal nen plus que sur une coite ». Michel Amiclon pendu, mains liées et en chemise, au gibet de Clisson le jeudi saint à deux heures et demi de l'après midi était encore en vie le vendredi à midi : tout ce temps il avait prié et s'était senti soutenu sous les pieds. En effet la Légende dorée rappelle la prière de la sainte à l'heure de sa décollation: "Ô Jésus [...] je vous en conjure, que quicquonque, en mémoire de mon martyre, m'invoquera à son heure dernière, [...] vous trouve propice et obtienne ce qu'il demande $~^{62}$. L'Anglais qui, las de traîner Jean Hurpois vers Alençon, décida de décapiter son captif plutôt que de subir ses plaintes et n'arriva pas en trois coups à lui entamer le col comprit alors que Catherine avait dû intervenir, il eut donc pitié du malheureux et songea sans doute au salut de son propre corps et de sa pauvre âme. La roue du supplice de la sainte n'avait-elle pas en éclatant tué quatre mille assistants. L'intervention protectrice a pu aller jusqu'à se manifester par « une voix » ou « une advision" prévenant - au début sans succès- le curé de Clisson d'aller décrocher Michel Amiclon. La voix s'adressa ensuite à Michel lui-même, dépendu mais encore captif, elle lui enjoignit d'abord de se dépêcher d'accomplir son voyage à Fierbois ${ }^{63}$, et elle réitéra le 23 avril, fête de saint Georges, jour des chevaliers qui devraient savoir tenir leur promesse, non sans rudesse : « "Autresfois, je t'avoye enchargié et commandé que tu allasses acquiter en ma chapelle de Fierbois ce que tu m'as promis, dont ne foys compte et pour ce delivre toy d'y aller hastivement sans plus tarder". Et ce dit la dite voix luy donna sur la joue un tres grand coup et luy fist mal $»^{64}$. Les compagnons de Michel ont bien vu la trace de la gifle...

15 L'enregistrement des récits sert de preuve aux miracles et surtout atteste de l'accomplissement du vœu de se rendre à Fierbois, ou d'y envoyer un représentant ${ }^{65}$. À partir du début du XVe siècle, des témoins viennent certifier par l'enregistrement de leur présence, non pas les événements auquel ils n'ont pas assisté, mais la réalité de la venue du narrateur à la chapelle, l'accomplissement de son voyage et le serment solennel qu'il a fait de l'authenticité de ce qui est arrivé. Les gardes de la chapelle sont le plus souvent ces témoins mais on trouve également des compagnons du déposant: Guillem de Cerville écuyer pour Alain le Roux, Jean Fardeau et Clément Levesque de la compagnie d'Alain Giron pour Guillem Guy et surtout un superbe trio pour le Génois Jean du Chastel : « monseigneur le bastard d'Orléans, le bastard de la Marche, la Hire et plusieurs autres $»^{66}$. 

les perspectives vers les tentatives d'évasion de Jeanne d'Arc. En juin 1428 Jean du Chastel, de la compagnie de Renault-Guillem de Vignolles frère de La Hire, était venu en belle escorte, on le voit, remercier sainte Catherine d'avoir été à ses côtés lors d'un duel qui devait régler le différend qui l'opposait à l'Anglais « d'Angleterre » Jean le Paintre sur les conditions de la capture du Génois devant Vendôme. Sorti victorieux du combat, du Chastel vint à la Chapelle de sainte Catherine « tout armé du harnois de son dit ennemy » et en fit don «à en faire mémoire perpétuel ». Il s'agit là de la première mention conservée du dépôt d'armes en ex-voto à Fierbois, et ce fut fait en présence de tout un groupe de chevaliers et combattants armagnacs, quatre mois avant le début du siège d'Orléans où se retrouvèrent ces mêmes chevaliers aux côtés de Jeanne d'Arc. Ce n'est d'ailleurs pas le seul ex-voto, en dehors des cierges, cité avant 1430 : l'Écossais Michel Amiclon apporta le licou où il fut suspendu, François Dye et Jean Gilbert offrirent leurs fers, et le "perdrieux " Jean de Pons Prins, plus prosaïquement, son gagne-pain, le matériel à prendre les perdrix, avec la corde qui ne l'avait pas pendu.

Jeanne a raconté être allée à Fierbois avant d'arriver à Chinon ${ }^{67}$, elle a sans doute vu les armes de l'Anglais, qui devaient être ostensiblement exposées, et on a dû lui raconter la raison de leur présence. Il est aussi vraisemblable que ses compagnons aient plus tard parlé avec elle des vertus particulières des prières à sainte Catherine et de l'efficacité toute spéciale du sanctuaire tourangeau. Je ne me hasarderai pas à prétendre que l'épée de Fierbois, plutôt que celle de Charles Martel, serait une arme gagnée sur un Anglais par un Génois! Ce n'est pas ici ce qui me soucie. En revanche, la façon dont les récits des essais de la Pucelle pour s'échapper de Beaulieu et Beaurevoir se rapprochent de fort près des textes conservés à Fierbois, c'est cela que je souhaite maintenant examiner.

18 Après avoir été capturée à Compiègne (le 23 mai 1430), Jeanne fut emmenée dès le 26 mai à Beaulieu, elle y demeura jusqu'au 10 juillet 1430. Et elle tenta dès lors de s'en échapper. Lors du procès on l'interrogea le 15 mars 1431 sur sa première tentative d'évasion, j'utilise ici le texte du manuscrit en français dit d'Urfé, pour sa parenté avec la langue du Livre des miracles ${ }^{68}$. D'abord les juges s'inquiètent de son désir de s'échapper, une fois prise. Une capture selon les règles du jeu des rançons supposait une entente verbale entre le preneur et le saisi, « rescous ou non rescous », et l'on fixait avec des serments en forme (la «foi donnée ») les modalités de versement et de libération du captif ${ }^{69}$. S'enfuir après avoir promis de rester était un parjure très grave, indigne de la noblesse combattante. Ces usages, évoqués dans le Livre des miracles lors de l'affaire de Jean Chastel, ne semblent pas avoir été suivis à Compiègne. Les questions que posèrent les juges et le récit que fit Jeanne portent sur l'annonce qu'avaient fait ses voix de sa capture, sur le lieu exact de la prise, l'étendard, le cheval, les armoiries, mais il n'y eut rien sur les conditions précises de la reddition. Sans promesse de rester sous le regard de son preneur, sans négociation de rançon où elle fût partie prenante, il n'était pas illicite ni indigne de la noblesse fraîchement acquise de ses frères, qu'elle cherchât à s'enfuir, et elle le dit sans ambages à ses juges: "elle ne fut oncques prisonniere qu'ele ne se eschappast voulentiers $»^{70}$.

19 La tentative de Beaulieu commence comme un miracle : Jeanne est sortie de la tour, tout simplement par une voie libre, comme les quatre de la Souterraine qui trouvèrent devant eux la porte ouverte ou encore l'homme d'Aunis devant lequel le pont-levis s'abaissa. Elle est sortie «entre ${ }^{71}$ ) deux pièces de bois ». La formule est sibylline, je 
pense qu'elle peut s'éclairer par les récits de Fierbois : Jeanne fut assez audacieuse pour sortir de la tour de Beaulieu avec des entraves, peut-être la taille et les bras pris entre deux ais, tout comme François Dye et Jean Gilbert qui partirent avec leurs fers et elle a voulu refermer une porte derrière elle, empêchant ainsi ses gardes de la suivre, ou même de s'apercevoir de sa fuite. Jusqu'ici, nous restons dans la droite ligne des textes de Fierbois et l'on peut penser que Jeanne s'y crut également car elle n'avait pas dû trouver d'obstacles immédiats. Elle ajoute quelques instants plus tard qu'elle pouvait bien tenter le coup, allegans illud proverbium in gallico vulgatum: "aide-toi, Dieu te aydera ${ }^{72}$ - ce qui lui sera reproché ensuite comme défi à Dieu. Et c'est là que le miracle n'a pas eu lieu. Alors que Guillem Bressin s'est échappé de Rouen «sans que ce que personne lui meffeist $\aleph^{73}$, que les poursuivants d'Alain Le Roux ne le virent pas ${ }^{74}$, le portier de Beaulieu non seulement vit Jeanne mais surtout s'interposa ${ }^{75}$, au lieu de la laisser aller comme les Anglais de Pierre de Saint-Jean ou de Jean Ducoudray, les flamands de Guillaume Oade, les Bourguignons de Perrin Gougeon. Le portier ne pouvait risquer sa vie en fermant les yeux sur la fuite d'une prisonnière bien sûr plus précieuse, tant en matière politique que de bon or trébuchant, que tous les miraculés de Fierbois réunis.

La deuxième tentative prit place lors de son séjour à Beaurevoir entre le 10 juillet et le 9 novembre 1430, elle fut plus sérieuse et son échec plus douloureux. Les raisons qu'elle invoque, lors de la séance du 3 mars 1431, pour avoir tenté de s'enfuir sont exactement celles que donnèrent Jean des Croix et ses parents : quand ils apprirent qu'ils allaient partir en Angleterre, ils ne purent le supporter et la veille du transport, prièrent afin de ne pas être menés en Angleterre et firent en sorte de se retrouver dehors ${ }^{76}$. Jeanne "quant elle sceut les Anglois venir, elle fut moult courroucee [...] et enfin pour la doubte des Anglois, sailli et se commanda a Dieu et a nostre Dame $»^{77}$. Dans le Livre des miracles le même verbe saillir est utilisé par le Gallois Oade qui « saillit » du faîte du toit de chaume en flamme d'un maison de Poperinghe où il s'était réfugié, et quelques minutes après, toujours poursuivi, "il lui convint saillir en une grant riviere et parfonde ${ }^{78}$ \%. Jeanne a donc délibérément accepté de sauter de haut. Dans un cas semblable les miraculés de Fierbois sentaient alors la protection immédiate de sainte Catherine. Jean du Coudray et Jean Courtin et leurs compagnons, malgré les cris du portier, montèrent sur les murs de Bellême hauts de deux lances, du Coudray fit passer par dessus les murailles le guetteur qui l'avait saisi, encouragea les fuyards en leur assurant " que madame sainte Katherine estoit a leur aide », et tous " se laisserent coler dessus le murs es doves ». Jeanne a également eu le réconfort de la sainte, mais après l'échec douloureux de sa tentative de Juillet $1430^{79}$. Catherine lui certifie la guérison des contusions de la chute et lui demande d'accepter son échec, en faisant bon visage à l'adversité. Le 14 mars 1431, Jeanne tout en reconnaissant son revers de Beaurevoir et qu'elle a dû en demander pardon à Dieu, insiste sur l'espérance qui la poussait et le manuscrit d'Urfé dans sa langue proche de celle utilisée par la jeune fille a cette expression superbe: "je le faisois non en esperance de moy deesperer, mais en esperance de sauver mon corps et de aller secourir plusieurs bonnes gens... ${ }^{80}$ ”, formule qui aurait pu être celle de l'écuyer André Escorneau sautant dans la nuit noire des rivières jusqu'alors à lui inconnues ${ }^{81}$. Les juges ne pouvaient dire que se confier à sainte Catherine pour s'échapper était fort aventuré et sans espoir! On disposait à Rouen de reliques venues directement du tombeau de la sainte, sa réputation était grande dans la ville même et chacun connaissant l'étymologie populaire de son nom de Catena la chaîne, celle qui délivre les gens aux fers ${ }^{82}$. Le 15 mars, les juges en interrogeant 
toujours Jeanne sur ses relations avec saint Michel et les deux saintes Catherine et Marguerite reviennent sur ses désobéissances aux conseils des voix, c'est que tout en ne voulant pas admettre que la Pucelle ait eu des révélations d'origine divine, il fallait cependant éclaircir les raisons de la défaillance de sainte Catherine à la libérer. Jeanne qui a accepté finalement d'assumer d'avoir enfreint délibérément les «mandements " de ses conseils, trouve cependant une fort belle parade qui redonne aux saintes leur pouvoir protecteur. Elle n'a pas pu s'empêcher de partir, la nécessité était trop forte, et les saintes ont été sensibles à cette exigence, non de se s'évader pour elle-même, mais pour aller secourir les gens de Compiègne. Elles sont donc intervenues et le Procès l'exprime par une redondance ambiguë « elles lui secoururent sa vie et la gardèrent de se tuer ${ }^{83}$ ». La première formule rappelle bien le soutien qui fait que le corps qui s'évade ne pèse pas tout son poids, mais les notaires n'en notent pas moins que le doute persiste sur la tentation du suicide. Et finalement c'est cette version qui fut reprise le 28 mars, refusant de considérer que la confiance en la protection de Catherine ait joué un rôle dans ce qu'on nommera par la suite le saut de Beaurevoir: "Comme une désespérée [elle] tenta de se précipiter d'une tour élevée [...] Elle se précipita, poussée et induite par un instinct diabolique ${ }^{84}$ ».

21 Un peu plus tard, en cette même journée du 28 mars, les juges, toujours inquiets des doutes qui pourraient désormais planer sur l'efficacité protectrice de la sainte du Sinaï envers ceux qui lui demanderaient secours, dans l'article XLVI remplacent une éventuelle prière de Jeanne à Catherine (que le texte n'a jamais mentionnée) pour l'aider à s'évader par une prière assurée de la jeune fille faite aux saintes avant de sauter pour que celles-ci intercèdent auprès de Dieu en faveur des gens de Compiègne : Item dixit se requisivisse multum affectuose sanctas Catherinam et Margaretam pro illis de Compendio, antequam se saltaret, dicens: "et comment laissera Dieu ainsi mourir mauvaisement ceulx de Compiengne qui sont si loyaulx $»^{85}$, prière qui ne se trouve pas du tout dans les pages antérieures du Procès.

Ainsi, et avant de complètement retourner en suicide, à l'instigation du diable ${ }^{86}$, le "saut de Beaurevoir ", les juges ont bien parlé d'une prière aux saintes Catherine et Marguerite accomplie par Jeanne avant de tenter de s'évader de la tour où elle était enfermée. La Pucelle, enfermée dans sa prison, ligotée, aux fers, maltraitée, voire harcelée par les soldats, ${ }^{87}$ rançonnée sans avoir discuté rançon lors de sa prise, se trouvait exactement dans la situation des soldats dont les récits de Fierbois, repris et commentés dans les compagnies de soldats, étaient certes plus agréables aux oreilles de Jeanne que les bravades de corps de garde. Les tentatives d'évasion qu'elle a faites me semblent correspondre aux circonstances les plus banales racontées dans le Livre des Miracles de sainte Katherine. Eut-elle réussi, nous aurions eu un récit supplémentaire, elle a échoué, comme sans doute beaucoup. Cet échec fut cruel pour elle, il la laissa aux mains de ses ennemis mortels. Mais il était aussi dangereux aux yeux des juges: il semblait nier le pouvoir protecteur général de la sainte, une sainte si révérée à Rouen, et ici peu importait en fait que ce fut vraiment Catherine qui ait été une des voix de Jeanne. Il fallait donc absolument transformer en désir de mort d'un cœur désespéré le saut vers la vie de Beaurevoir. 


\section{NOTES}

1. J. Quicherat, Aperçus nouveaux sur l'histoire de Jeanne d'Arc, Paris, 1850. P.- H. Dunand, Études historiques sur les voix et visions de Jeanne d'arc, Paris, 1903, 2 vol.

2. E. Lucie-Smith, Joan of Arc, Londres, 1976 et sa discussion par G. Van den Aardweg, "Une réhabilitation psychologique : Jeanne d'Arc ", Communio, VIII-1, 1983, pp. 80-91.

3. Méliès a dû tourner Jeanne d'Arc en 1897-1898, mais on ne sait si le film précède réellement ou non le Jeanne d'Arc de Hatot (1898). Le film récent est celui de Besson (The Messenger, 1999).

4. Livre des miracles de Sainte-Catherine-de-Fierbois, éd. Y. Chauvin, Poitiers, Société Historique du Poitou, 1976 (Archives Historiques du Poitou, LX). Édition partielle - et non recommandable - de l'abbé J. Bourassé, Les miracles de madame Sainte Katherine de Fierboys en Touraine, Paris, 1858. Outre l'infidélité des transcriptions, ce recueil est remarquable par le choix de miracles qui concernent uniquement les gens d'armes, sans que le chanoine et Président de la plus qu'honorable société Archéologique de Touraine se soit expliqué sur cette sélection.

5. On consultera à mon avis avec profit: K. Sullivan, "II do not name to you the voice of st Michel" : The identification of Joan of Arc's voices ", Fresh verdicts on Joan of Arc, éd. B. Wheeler et C. T. Wood, New York, Garland, 1996, pp. 85-111.

6. P. Tisset, Y. Lanhers éd., Procès de condamnation de Jeanne d'Arc, Paris, Klincksieck, (Société de l'Histoire de France), 1960-1971, t. 1, p. 71 (texte latin). Plus loin cité comme : Procès condamnation. 7. Ego non habeo de hoc dicendo licentiam, Procès condamnation, I, p. 71. Profitons du discours direct, si rare dans le procès et osons nous détacher de la transcription de la minute française (manuscrit dit d'Orléans) : « je n'ai pas congé de vous le dire ».

8. Procès de condamnation, I, p. 107 ( 3 mars 1431). La minute française use de l'expression aujourd'hui déviée de son sens médiéval : « qu'elle fit bonne chiere » (ibid.).

9. Sainte-Catherine de Fierbois, malgré des tentatives antérieures ne devint paroisse autonome qu'en 1704. (Act. com., c. Sainte-Maure, ar. Tours, Indre-et-Loire).

10. Livre des Miracles, p. 4.

11. Id., 4, p. 2 : Fouquault de la Rivière de Nevers en Angoumois, (soit Asnière-sur-Nouère en Charente, selon l'éditeur).

12. Id., 5, p. 2 : Les parents d'un enfant d'Angers réchappé d'une chute dans un puits viennent remercier sainte Catherine le $1^{\mathrm{er}}$ juin 1379.

13. Miracle de la femme d'Hilaire Hubert, de la paroisse voisine de Saint-Épain, paralysée pour avoir souhaité que son mari restât dans cette chapelle qu'il restaurait au détriment de « besogne » qu'elle jugeait plus urgente.C'est elle qui accomplit le vœu de son époux en allant à la fameuse chapelle après sa propre guérison! (Id., 6, pp. 2-3).

14. Livre des miracles, 28, p. 10.

15. Les Anglais étaient logés au château de Chamant, près de Senlis et le miraculé était de SaintSauveur de Luzarches à une quinzaine de kilomètres au sud-ouest de cette ville, la cohérence des lieux est parfaite.

16. A. Poncelet (éd.), « Sanctee Catarince et miracula Rotomagensis », Annalecta Bollandiana, XXII, 1903, pp. 423-438.

17. L. Musset, "Les translations de reliques en Normandie (IX ${ }^{\mathrm{e}}-\mathrm{XII}{ }^{\mathrm{e}}$ siècles) », Les saints de la Normandie médiévale, Colloque de Cerisy-la-Salle (26-29 octobre 1996), P. Rouet et F. Neveux éd., Caen, Presses Universitaires de Caen, 2000, p. 107.

18. Jacques de Voragine, La Légende dorée, J.-B. M. Roze trad., Paris, Garnier-Flammarion, 1967, t. II, p. 392.

19. Catherine se retrouve parmi les saints et saintes protecteurs dans cinq confréries de la ville de Rouen et elle figure soixante huit fois dans les confréries de la province (loin derrière sainte 
Barbe...) (cf. C. Vincent, Des charités bien ordonnées, les confréries normandes de la fin du XIII ${ }^{e}$ siècle au début du XVI ${ }^{e}$ siècle, Paris, 1988, tableau p. 303)

20. C. A. Lewis, "Model girls", virgins, martyrs and the training of young women in late medieval England ", Young medieval women, Thrupp Stroud, Sutton publishing, 1999, pp. 24-46.

21. Sur les douze livres d'heures sortis d'ateliers d'Avignon entre 1375 et 1420, trois comportent un office de sainte Catherine (M.-C. Leonelli, « La dévotion aux saints d'après les livres d'heures confectionnés à Avignon », Le peuple des saints. Croyances et dévotions en Provence et Comtat Venaissin des origines à la fin du Moyen Âge, Mémoires de l'Académie de Vaucluse, $7^{\mathrm{e}}$ série, VI, 1985, p. 230.).

22. Un rapide sondage dans les catalogues d'exposition: L'art au temps des rois Maudits Philippe le Bel et ses fils (1285-1328), Paris, 1998, et Les fastes du Gothique, Paris, 1981 montre que de l'un à l'autre au cours du XIV ${ }^{\mathrm{e}}$ siècle les commandes princières de représentation de la sainte se sont fortement accrues, peut-être avec le retour de la guerre (cf. Les fastes... notices des $n^{\circ}: 22,77.215$, $219,235,315)$. La sainte figure dans $30 \%$ des retables à prix faits notariés de Provence ente 1390 et 1400 et dans $40 \%$ d'entre eux entre 1420 et 1430 (cf. D. Gidrol-Antonelli, Saint honorés en Provence d'après les prix faits des retables, Le peuple des saints, op. cit., p. 361).

23. Il aurait selon, sa biographie fort romancée, offert ses services au sultan (Le livre des faits du bon messire Jean de Boucicaut dit Le Meingre et gouverneur de Jennes, D. Lalande éd., Genève, Droz («Textes Littéraires français », 331).

24. D. Lalande, Jean II le Meingre, dit Boucicaut (1366-1421) : étude d'une biographie hérö̈que, Genève, Droz, (Publications romanes et Françaises, 184), 1988 ; J. Delaville le Roulx, La France en Orient au XIVe siècle: expéditions du maréchal de Boucicaut, Paris (Bibliothèque des Écoles françaises d'Athènes et de Rome 44-45), 1886.

25. N. Housley, "Le Maréchal de Boucicaut à Nicopolis ", "Nicopolis, 1396-1996", Annales de Bourgognes, 68-3, 1996, pp. 85-99.

26. Le bref du pape est cité par J.-B. Fourault, Sainte-Catherine de Fierbois, ses monuments et ses souvenirs de Jeanne d'Arc. Notice historique et archéologique, Tours, 1877, p. 15.

27. Livre des miracles, 86, p. 40 (17 juillet 1420) : Jean des Croix, son frère et son neveu plus un écuyer prisonniers à Verneuil, libérés juste avant d'être envoyés en Angleterre.

28. "Casin du Boys, ung gentilhomme frequentant les armes", pris par les Bourguignons à Beaumont-sur-Oise en 1418 (Id., 92, p. 46).

29. Aimeri de Lusignan, (Id., 61, p. 27, 1407), Guillaume Guy (Id., 94, p. 48, 1421), André Estorneau (Id., 107, p. 43, 1430).

30. Id., 102, p. 54 (avril 1426), François Dye et Jehan Gilbert « compaignons de la garnison de Beaumont de la Ronce ".

31. Id., 104, p. 55 (11 juin 1428) Jehan du Chastel, genevois (ou génois ?), de la compagnie de Renaud-Guillem de Vignolles (frère d'Étienne dit La Hire).

32. Id., 105, p. 57 (4 mai 1429) Michel Amiclon de la compagnie de Jean Stuart.

33. Id., 75, p. 35.

34. Id., 57, p. 25 (26 mars 1383) et 71 p. 32 (même date).

35. Id., 103, p. 55 [1426].

36. Id., 29, p. 11 (Chalucet) ; 60, p. 27 (Falaise) ; 86, p. 40 (Verneuil) ; 96, p. 60 (Bellême) ; 101, p. 53 (Alençon) ; 102, p. 54 (La Châtre-sur-le-Loir).

37. Id., 92, p. 46 (Beaumont-sur-Oise); 89, p. 43 (Corbeil); 105, p. 57 (Clisson); 58, p. 25 (Poperinghe) ; 95, p. 49 (près de Meunet-Berry).

38. Id., 57, p. 25 et 71, p. 32.

39. Id., 59, p. 27.

40. Id., 78, p. 36 .

41. Id., 89, p. 43.

42. Id., 94, p. 48.

43. Id., 97, p. 41. 
44. Id., 78, p. 36 .

45. Id., 96, p. 50.

46. Id., 94, p. 48, c'est ce qui lui vaut de se retrouver dans un tonneau.

47. Id., 59, p. 27, dans la citerne déjà évoquée, quelque part en Savoie !

48. Id., 99, p. 52 (13 janvier 1427 n. st.) : c'est du moins ainsi que le texte les présente d'abord, au cours du récit on apprend incidemment que Courtin est enfermé depuis la journée de Verneuil (17 août 1424) ce qui éclaire d'un jour nouveau les raisons de sa capture.

49. Id., 100, p. 52 .

50. Id., 105, p. 57.

51. Id., 108, p. 63.

52. Id., 52, p. 22. Cet homme de Périgueux a été par deux fois sauvé de la mort par l'intervention de sainte Catherine et vint à Fierbois en mars 1401 (n. St.). (Id., p 53, p. 23).

53. Id., 86, p. 40 : « Très chiere dame plaise toy, de ta benigne grace, nous ayder et conforter et prier Dieu pour nous... »

54. Id., 75, p. 35.

55. Id., 60, p. 27. Ce sont trois hommes de Gémigny près d'Orléans, prisonniers à Falaise, on ne sait trop pourquoi.

56. Id., 97, p. 50 (avril 1423). On ne sait si Moricet a accompli son vœu !

57. Id., 78, p. 36.

58. Id., 102, p. 54 : « si attachèrent leurs fers le plus haut haut qu'ils peurent ».

59. Id., 99, p. 52. On doit comprendre que les uns ont des entraves métalliques et les autres les pieds pris entre deux barres de bois maintenues par des cadenas.

60. Id., 103, p. 55 [juin 1426]. Pris alors qu'il faisait charger du foin, emmené, ligoté à un lit, cet homme de Montreuil-Bellay s'est trouvé délié après son vœu tandis que ses geôliers s'endormaient.

61. Id., 95, p. 49 : « et ne sentoit nulle angoesse ».

62. La Légende dorée, op. cit., II, p. 392.

63. Id., 105, p. 56 : la nuit du dimanche au lundi de Pâques : «Délivre-toi, délivre-toi, si pense d'aler acomplir en ma chapelle de Fierbois ce que tu as promis, et je te garderay et aideray ».

64. Ibid.

65. Id., 94, p. 48 : C'est Thomas Bonvallet, écuyer de la compagne d'Alain Giren qui fit le récit de la délivrance de son camarade Guillaume Guy.

66. Id., 104, p. 57.

67. Si l'on prend comme date de départ de Vaucouleurs le samedi (12 février veille du dimanche «des Bures »), cette visite aurait pris place le 22 février 1429. Elle raconta le 22 février 1431 ( $2^{\mathrm{e}}$ séance du Procès) qu'elle envoya prévenir le roi de son arrivée lors de ce passage à Fierbois (misit ad illum quem dicit regem suum,on notera la restriction des scribes sur son roi), et ajouta lors de la séance suivante ( 3 février) qu'elle y avait entendu trois messes dans la journée et précisa que son message au roi était écrit (misit litteras).

68. Tisset-Lanhers, Procès de condamnation, t. I, p. 155.

69. J. Glénisson, V. Deodato da Silva, La pratique et le rituel de la reddition aux XIV et $X V^{e}$ siècles, Jeanne d'Arc, une époque, un rayonnement, Colloque d'histoire médiévale Orléans, octobre 1979, Paris, C. N. R. S., 1979, p. 113-122.

70. Ibid.

71. Inter selon P. Champion et Tisset, intra selon Quicherat.

72. Procès, I, p. 156. En français dans le texte latin.

73. Livre des miracles, 96, p. 50.

74. Id., 103, p. 55.

75. Procès, I, p. 155 : « N'eust été le portier qui la advisa et la recontra »; nisi portarius qui eam vidit et sibi obviavit. 
76. Livre des miracles, 86, p. 40 (1420).

77. Procès, I, p. 107.

78. Livre des miracles, 58, p. 26.

79. La date précise en est inconnue, certains auteurs pensent que ce fut vers le 20 juillet car Jean de Luxembourg aurait été prévenu d'avoir à livrer Jeanne aux Anglais vers le 14 juillet. La hauteur dont elle se jeta varie selon les commentateurs de $24 \mathrm{~m}$ à moins de $6 \mathrm{~m}$ !

80. Procès, I, p. 153.

81. "Si chevaucha en traversant pais, sans tenir chemin ne sentier, toute la nuyt en s'en retournant et oncques ne faillit a son chemin, et passa plusieurs rivieres moult fortes a passer et oncques n'y avoit esté ", Livre des miracles, 107, p. 62.

82. Cette étymologie catena est donnée mais immédiatement discutée par la Légende dorée qui préfère y voir l'image de l'inflexibilité de la sainte.

83. Procès, I, p. 161 : ipse succurerunt vite eius et preservaverunt eam ipsam interficiret.

84. Procès, I, p. 242.

85. Procès, I, p. 246. Jeanne répondit qu'on avait à se référer à ce qu'elle avait déjà dit.

86. Procès I, p. 282 : Principalement au dit saut de la tour de Beaurevoir il apparaît manifestement qu'elle a tenté Dieu (tentasse Deus manifeste videtur).

87. C'est à Beaurevoir, on ne sait exactement quand, que le chevalier Raimond de Macy a tenté plusieurs fois de "peloter» Jeanne. Que l'on me pardonne cette expression la seule qui me paraisse traduire : "temptavit [...] cum ea ludendo tangere mammas suas intendo ponere mnus in sinu suo » (P. Duparc, Procès en nullité de la condamnnation de Jeanne d'Arc, Paris, Klincksieck, Paris, 1977, t. I, p. 405).

\section{AUTEUR}

\section{FRANÇOISE MICHAUD-FRÉJAVILLE}

Université d'Orléans 